\title{
RESEARCH
}

Open Access

\section{The Inc-CTSLP8 upregulates CTSL1 as a competitive endogenous RNA and promotes ovarian cancer metastasis}

Xinjing Wang ${ }^{1 \dagger}$, Xiaoduan $\mathrm{Li}^{2 \dagger}$, Feikai Lin ${ }^{1}$, Huizhen Sun ${ }^{1}$, Yingying $\mathrm{Lin}^{3}$, Ziliang Wang ${ }^{1 *}$ and Xipeng Wang ${ }^{1 *}$

\begin{abstract}
Background: Ovarian cancer is highly lethal and has a poor prognosis due to metastasis. Long non-coding RNAs (IncRNAs) are key regulators of tumor development, but their role in ovarian cancer metastasis remains unclear.

Methods: The expression of Inc-CTSLP8 in ovarian cancer was analyzed in public databases (TCGA and GEO) and validated via qRT-PCR. Lnc-CTSLP8 overexpression and knockout cell lines were constructed using a lentiviral vector and the CRISP/Cas9 system. Cell proliferation, colony formation, migration, and invasion were analyzed. An ovarian orthotopic tumor mouse model was used for the in vivo study. Changes in autophagosomes, autolysosomes, and mitochondria in ovarian cancer cells were observed via transmission electron microscopy. EMT markers were detected by immunoblotting and immunofluorescence assays. RNA immunoprecipitation, RNA pull-down, and dual luciferase reporter assays were performed to confirm the interaction between Inc-CTSLP8 and miR-199a-5p.

Results: A novel pseudogene, Inc-CTSLP8, was identified in ovarian cancer, with significantly elevated expression in metastatic tumor tissues compared to primary ovarian tumors. When overexpressed, Inc-CTSLP8 promoted ovarian cancer in vitro and in vivo by acting as a sponge for miR-199a-5p. Autophagy and EMT in ovarian cancer were also enhanced by Inc-CTSLP8. Mechanistically, Inc-CTSLP8 upregulated CTSL1 as a competitive endogenous RNA and exhibited oncogenic effects. Moreover, CTSL1 inhibitor treatment and miR-199a-5p overexpression abrogated the effects of Inc-CTSLP8 overexpression.
\end{abstract}

Conclusions: Inc-CTSLP8 acts as a ceRNA in ovarian cancer and represents a potential therapeutic target for metastatic ovarian cancer.

Keywords: Lnc-CTSLP8, Ovarian cancer, ceRNA, CTSL1, Metastasis

\footnotetext{
* Correspondence: wangziliang@xinhuamed.com.cn;

wangxipeng@xinhuamed.com.cn

${ }^{+}$Xinjing Wang and Xiaoduan Li contributed equally to this paper and should

be considered as co-first authors.

'Department of Gynecology and Obstetrics, XinHua Hospital, Shanghai

JiaoTong University School of Medicine, 1665 Kongjiang Rd, Yangpu District,

Shanghai 200092, China

Full list of author information is available at the end of the article
}

(C) The Author(s). 2021 Open Access This article is licensed under a Creative Commons Attribution 4.0 International License, which permits use, sharing, adaptation, distribution and reproduction in any medium or format, as long as you give appropriate credit to the original author(s) and the source, provide a link to the Creative Commons licence, and indicate if changes were made. The images or other third party material in this article are included in the article's Creative Commons licence, unless indicated otherwise in a credit line to the material. If material is not included in the article's Creative Commons licence and your intended use is not permitted by statutory regulation or exceeds the permitted use, you will need to obtain permission directly from the copyright holder. To view a copy of this licence, visit http://creativecommons.org/licenses/by/4.0/ The Creative Commons Public Domain Dedication waiver (http://creativecommons.org/publicdomain/zero/1.0/) applies to the data made available in this article, unless otherwise stated in a credit line to the data. 


\section{Background}

Ovarian cancer is the fifth leading cancer-related cause of death in women, with the highest mortality rate among gynecological malignancies [1]. In China, over 52,000 new ovarian cancer patients are diagnosed, and 22,500 patients die from ovarian cancer each year [2]. Early diagnosis is difficult due to the lack of established biomarkers and specific clinical symptoms, leading to extremely poor prognosis $[3,4]$. More than $50 \%$ of patients are diagnosed at an advanced stage with widespread metastasis [5]. Therefore, novel biomarkers for early diagnosis and therapeutic targets for ovarian cancer metastasis are of urgent necessity.

Non-coding RNAs (ncRNAs) play an important role in various diseases, including ovarian cancer $[6,7]$. Our previous study showed that distinct transcriptome patterns of monocytes, including the ncRNA expression profile, were potentially associated with tumor angiogenesis in ovarian cancer [8]. In addition, ncRNAs such as miRNAs could be transferred via exosomes and suppress $\mathrm{T}$-cell differentiation within the ovarian cancer microenvironment, leading to tumor progression [9]. Recently, intricate interactions among different RNA types have been reported and described as competing endogenous RNA (ceRNA) networks, which include mRNAs and non-coding RNAs such as long non-coding RNAs (lncRNAs) [10, 11]. Further, IncRNAs have been shown to play important roles in tumorigenesis and metastasis through their function as ceRNAs [12, 13].

Pseudogenes are a subclass of lncRNAs that share high sequence homology with protein-coding genes without the ability to encode proteins. Importantly, pseudogenes participate in the post-transcriptional regulation of their protein-coding counterparts $[14,15]$. For example, PTEN pseudogene 1 (PTENP1) acts as a decoy against PTEN-targeting miRNAs with tumor suppressor activity [16]. Similarly, BRAF pseudogene 1 (BRAF1) upregulated its cognate BRAF by competitively interacting with shared miRNAs, promoting lymphoma [17]. However, pseudogenes in ovarian cancer are yet to be investigated, which might lead to a better understanding of the role of non-coding RNAs in the disease.

Cysteine cathepsin L (CTSL1) is an important member of the cathepsin family and contributes to tumor progression through different mechanisms, such as the induction protein degradation and autophagy [18]. Moreover, CTSL1 enhances the epithelial-tomesenchymal transition (EMT) and promotes cancer invasion and metastasis $[19,20]$. In ovarian cancer, CTSL1 has been reported as an independent diagnostic and prognostic factor [21]. Moreover, elevated CTSL1 expression was associated with chemoresistance and metastasis of ovarian cancer [22].
In this study, we screened RNA expression patterns in metastatic ovarian cancer tissues via RNA sequencing and identified non-coding CTSL pseudogene 8 (lncCTSLP8), which was significantly upregulated. We then established lnc-CTSLP8 overexpression and knockout cell lines to investigate the role of lnc-CTSLP8 in ovarian cancer cells in vitro and in vivo. Mechanistically, we found that lnc-CTSLP8 upregulated its cognate gene CTSL1 by acting as a sponge for miR-199a-5p, and CTSL1 inhibition abrogated the oncogenic effects of lncCTSLP8.

\section{Methods}

\section{Patients and specimens}

A total of 76 benign ovarian cyst tissues, 219 epithelial ovarian cancer tissues, and 126 peritoneal metastasis ovarian cancer tissues, together with 38 serum and 38 ascites samples from the same cohort of cancer patients, were collected from benign ovarian cyst or epithelial ovarian cancer patients between 2008 and 2018, after informed consent had been obtained. The histopathological diagnosis, stage, and grade of ovarian cancer were based on the FIGO classification. Overall survival (OS) was defined as the time from the initiation of surgery to death of any cause or the most recent follow-up. The clinical data of ovarian cancer patients are shown in Table S1. This study was approved by the Ethics Committee of the Xinhua Hospital.

\section{TCGA and GEO data analysis}

The lncRNA-seq data were obtained from the Cancer Genome Atlas (TCGA) ovarian cancer cohort of 379 ovarian serous cystadenocarcinoma patients. The expression of lnc-CTSLP8 was normalized by variancestabilizing transformation (VST) and compared between patients of different stage and grade. The microarray data derived from dataset GSE19829 (https://www.ncbi. nlm.nih.gov/geo/query/acc.cgi?acc=GSE19829) of 28 epithelial ovarian cancer patients were used to analyze the correlation between CTSL1 and lnc-CTSLP8 expression.

\section{Immunohistochemistry (IHC)}

Tissue microarrays (TMAs) were conducted using benign ovarian cyst tissues, epithelial ovarian cancer tissues, as well as peritoneal metastasis ovarian cancer tissues and a CTSL1 antibody (ab203028, Abcam, 1:100 dilution). The intensity of staining was classified as weak, moderate, and strong. The IHC score was calculated as $\sum(\mathrm{PI} \times \mathrm{I})=($ percentage of cells of weak intensity $\times 1)+$ (percentage of cells of moderate intensity $\times 2$ ) + (percentage of cells of strong intensity $\times 3$ ). 


\section{Elisa}

Soluble CTSL1 protein levels in the serum and ascites of ovarian cancer patients were detected via ELISA using the Human Cathepsin L DuoSet ELISA Kit (R\&D Systems, USA).

\section{Cell culture}

Human ovarian cancer cell lines were purchased from the Cell Bank of the Chinese Academy of Sciences and cultured in RPMI-1640 medium (Gibco, USA) supplemented with $10 \%$ fetal bovine serum (FBS, Gibco, USA) and $1 \%$ antibiotics. SKOV3, OVCA420, and Hey cell lines were transfected with a luciferase reporter vector containing a neomycin resistance gene and cultured in complete medium supplemented with $50 \mu \mathrm{g} / \mathrm{ml}$ neomycin. All cell lines were authenticated using short tandem repeat (STR) analysis.

\section{Lnc-CTSLP8 overexpression and knockout cell lines}

The human wild-type lnc-CTSLP8 lentiviral vector was designed by Genomeditech (China) and used to infect SKOV3 and OVCA420 cell lines in order to generate lnc-CTSLP8 overexpression cell lines SKOV3-CTSLP8-OE and OVCA420-CTSLP8-OE, respectively. Negative control cell lines were generated via infection with control lentivirus containing a random sequence (control vectors), and wild-type cells were used as blank controls.

To knock out lnc-CTSLP8 in ovarian cancer cells, eight sgRNAs were designed (Fig. S1B), and the sgRNA sequences are listed in Table S2. After infection and testing, sgRNA2 and sgRNA6 were selected, and a twogRNA-expressing plasmid was constructed for further lnc-CTSLP8 knockout (Fig. S1C). The Hey cell line was infected with lentiCas9-Blasticidin lentivirus and selected with blasticidin $(10 \mu \mathrm{g} / \mathrm{ml})$. Surviving cells were infected with the H_CTSLP8 (CTSL1P8) sgRNA (PGMLV-GM1) lentivirus and selected with puromycin $(2 \mu \mathrm{g} / \mathrm{ml})$. After selection, the cells were maintained with blasticidin $(5 \mu \mathrm{g} / \mathrm{ml})$ and puromycin $(1 \mu \mathrm{g} / \mathrm{ml})$. The isolated single clone was subjected to PCR and DNA sequencing for knockout validation. Sanger sequencing confirmed the deletion of CTSLP8 in the knockout single clone (Fig. S1D). Sanger sequencing data are provided as supplementary material (16305_T01C-14798CF1_10778P190923108A_H01.ab1).

The Hey-CTSLP8-KO cell line was used for further experiments. The negative control cell line was generated via infection of Hey cells with control lentivirus containing a random sequence that did not target any genes (control vector), and wild-type Hey cells were used as blank controls.

\section{RNA sequencing}

Ovarian cancer cell lines (SKOV3-CTSLP8-OE and negative controls) were used for RNA sequencing. Total RNA was extracted using the miRNeasy Micro Kit (Qiagen, Germany). Next-generation libraries were then prepared using the VAHTS mRNA-seq v2 Library Prep Kit or the VAHTS TM Total RNA-seq Library Prep Kit (Vazyme, China). Thereafter, RNA-seq libraries were sequenced using the HiSeq X10 system (Illumina, USA). Significantly differentially expressed genes were defined as those with a fold-change $>2$ and $p<0.05$. Gene Set Enrichment Analysis (GSEA) was used for gene function annotation.

\section{Single-cell RNA-sequencing}

Fresh ovarian cancer tissue, adjacent ovarian tissue, and peritoneal metastasis tissue samples were cut into small pieces and then enzymatically digested using the MACS tumor dissociation kit (Miltenyi Biotec, Germany). The cells were filtered using a 70-mm Cell-Strainer (BD Biosciences, USA) and centrifuged at $1200 \mathrm{rpm}$ for $5 \mathrm{~min}$. The pelleted cells were suspended in red blood cell lysis buffer (Miltenyi Biotec, Germany) in order to lyse red blood cells. For droplet-based single-cell RNA sequencing, single cells were processed using the GemCode Gel Bead, Chip, and Library Kit (10x Genomics, USA). A total of 6000 cells were loaded into each sample. Libraries were sequenced using the NovaSeq 6000 System. Single-cell RNA sequencing data were aligned and quantified using Cell Ranger Single-Cell Software Suite 2.3 (10x Genomics, USA).

\section{Quantitative real-time PCR}

Total RNA was extracted from cells or tissues with TRIzol (Invitrogen, USA) and subjected to PCR using the PrimeScript RT-PCR kit (Takara, Japan). The primers for IncRNAs and mRNAs are listed in Table S3. The miRNA was reverse-transcribed into cDNA using the miScript II RT Kit (Qiagen, Germany) and subjected to PCR using the miScript SYBR Green PCR Kit (Qiagen, Germany). The miRNA primers for has-miR-199a-5p were purchased from GenePharma (Shanghai, China). Relative gene expression was calculated using the comparative threshold cycle method.

\section{Immunofluorescence assay (IF)}

Cells or tissues were fixed with $4 \%$ paraformaldehyde and permeabilized with $0.3 \%$ Triton X-100. Next, the sections were incubated with $5 \%$ goat serum (Life Technologies, USA) for $1 \mathrm{~h}$ at room temperature, followed by incubation with primary and secondary antibodies. After staining with DAPI (Life Technologies, USA), the sections were imaged under a Leica SP5 confocal 
fluorescence microscope. The primary antibodies used are listed in Table S4.

\section{Western blotting}

Tissues or cells were lysed in RIPA buffer, and lysates were separated via $10 \%$ SDS-PAGE and transferred onto polyvinylidene fluoride membranes (Millipore, USA). The membranes were blocked with 5\% BSA for $2 \mathrm{~h}$, incubated with the primary antibodies listed in Table S4, and then incubated with secondary HRP-conjugated antibodies at room temperature for $1 \mathrm{~h}$. A chemiluminescent substrate (Millipore, US) was used to visualize the signals.

\section{Luciferase assay}

HEK293T cells were co-transfected with $100 \mathrm{ng}$ of CTSL1-3'UTR-Luciferase/mut CTSL1-3'UTR-Luciferase plasmids or CTSLP8-Luciferase/mut CTSLP8-Luciferase and predicted miRNAs/miR-NC (negative control), and $20 \mathrm{ng}$ Renilla plasmid was co-transfected to determine relative luciferase activity as the ratio of Firefly luciferase activity to Renilla luciferase activity. Luciferase activity was measured using a dual luciferase reporter system (Promega, USA) $48 \mathrm{~h}$ after transfection.

\section{RNA pull-down assay}

Biotinylated miR-199a-5p (5'-CCCAGUGUUCAGAC UACCUGUUC-3') or its mutant (5'-CUGUGACCUCAGACUACCUGUUC-3') were transfected into SKOV3-CTSLP8-OE cells. The cells were harvested, lysed, sonicated, and incubated with $\mathrm{C}^{-1}$ magnetic beads (Thermo Fisher Scientific, USA) at $4{ }^{\circ} \mathrm{C}$ for $3 \mathrm{~h}$. The RNA mix bound to the beads was then eluted and extracted with TRIzol (Invitrogen, USA) for qRT-PCR.

\section{RNA immunoprecipitation assay}

An RNA immunoprecipitation assay (RIP) was carried out in SKOV3 cells using a Magna RIP RNA-Binding Protein Immunoprecipitation Kit (Millipore, USA) according to the manufacturer's instructions. Argonaute2 (AGO2) antibody (Abcam, cat. \#ab186733) and immunoglobulin G (IgG) antibody (Millipore, USA) were used for the RIP assays, and the purified RNAs were extracted and analyzed via qRT-PCR. The primers used are shown in Table S3.

\section{CCK8 assay}

Ovarian cancer cells were seeded into 96-well plates (1500 cells/well), and CCK8 solution (Beyotime, China) was then added to each well daily for a total of 5 days. After incubation for $2 \mathrm{~h}$ at $37^{\circ} \mathrm{C}$, the absorbance at 450 $\mathrm{nm}$ was measured using a microplate reader (Thermo Labsystems, Finland). Cathepsin L Inhibitor Z-FY-DMK (Sigma, USA) was dissolved in DMSO at $20 \mu \mathrm{mol} / \mathrm{L}$ and added to the medium to obtain a final concentration of $1 \mu \mathrm{mol} / \mathrm{L}$. The cells were treated with Z-FY-DMK for a total of 5 days. After incubation with CCK8 solution, the absorption at $450 \mathrm{~nm}$ was measured. DMSO was used as the negative control.

\section{Colony formation assay}

Ovarian cancer cells were seeded in 6-well plates at 500 cells/well and cultured for 10 days. The colonies were stained with $0.2 \%$ crystal violet for $10 \mathrm{~min}$ and then counted.

\section{Migration and invasion assay}

A total of $6 \times 10^{4}$ cells were suspended in serum-free DMEM and added to the upper chamber of a Transwell chamber with $8-\mu \mathrm{m}$ pores (Corning, USA) (for migration assays) or a chamber pre-coated with Matrigel (BD Biosciences, USA) (for invasion assays). DMEM supplemented with $10 \%$ FBS was added to the lower chamber. After $24 \mathrm{~h}$, the cells that migrated or invaded the lower chamber were stained with $0.2 \%$ crystal violet and counted.

\section{Transmission electron microscopy (TEM)}

Ovarian cancer cells were fixed in fixing solution (Servicebio, China) at $4{ }^{\circ} \mathrm{C}$ for $4 \mathrm{~h}$ and post-fixed with $1 \%$ $\mathrm{OsO} 4$ for $2 \mathrm{~h}$ at room temperature, followed by dehydration and infiltration. The cells were then embedded, cut into ultrathin sections $(60-80 \mathrm{~nm})$, and observed under a transmission electron microscope (HITACHI, Japan).

\section{Animal tumor model}

Animal experiments were approved by the Animal Care and Use Committee of Xinhua Hospital. Female athymic nude mice (6 weeks old) were purchased from Slac Laboratory Animal Center (Shanghai, China) and randomly divided into different groups $(n=5)$ for ovarian orthotopic injection with $5 \times 10^{5}$ SKOV3-CTSLP8-OE, OVCA420-CTSLP8-OE, Hey-CTSLP8-KO cells, or control vectors and wild-type cell lines as negative and blank controls, respectively. Ovarian cancer cells in $10 \mu \mathrm{L}$ serum-free RPMI-1640 were injected into the left ovarian parenchyma of nude mice. In addition, Z-FYDMK $(100 \mathrm{mg} / \mathrm{kg})$ was intravenously injected through the tail vein each week, and DMSO was used as a negative control.

Two weeks after ovarian orthotopic injection, the tumor-bearing mice were examined for luciferin expression using D-luciferin $(100 \mathrm{mg} / \mathrm{kg}$, Invitrogen, US) to assess tumor development every week. After 6 weeks, tumors in each group were harvested following euthanasia, and the weights of tumors and metastasis organs were recorded. For survival analysis, 12 mice from each group were used to calculate OS from the ovarian 
orthotopic injection of different cell lines to death or to 12 weeks after ovarian orthotopic injection.

\section{RNA fluorescence in situ hybridization (FISH)}

To detect lnc-CTSLP8 expression in ovarian cancer cells and tissues, a lnc-CTSLP8 probe (5'-CY3-GGTTTTAA CCTGATCCTTCACAGGACTCAT-3') was designed and synthesized. The probe signal was detected using a FISH Kit (Servicebio, China) as per the manufacturer's instructions.

\section{Statistical analysis}

Data are presented as mean \pm SEM and were analyzed using SPSS 20.0 software (SPSS Inc., Chicago, IL, USA). Student's $t$-test, two-way ANOVA, and chi-square tests were used for comparison. Overall survival (OS) was calculated using the Kaplan-Meier method. Statistical significance was set at $p<0.05$.

\section{Results}

High expression of CTSL pseudogene 8 in ovarian cancer

Based on lncRNA-seq data derived from the TCGA ovarian cancer cohort of 379 patients, lnc-CTLSP8 was significantly elevated in advanced-stage and high-grade ovarian cancer samples (Fig. 1a, b). In addition, through qRT-PCR, we found that lnc-CTSLP8 expression was significantly higher in ovarian cancer tumor tissues $(n=$ 22) than in benign ovarian cyst tissues $(n=22)$. Further, lnc-CTSLP8 expression in ovarian cancer peritoneal metastasis tissues $(n=22)$ was even higher than that in ovarian cancer in situ tumor tissues (Fig. 1c).

\section{Lnc-CTSLP8 promotes the proliferation, migration, and invasion of ovarian cancer cells}

To assess the role of lnc-CTSLP8 in ovarian cancer, lncCTSLP8 expression in different ovarian cancer cell lines was assessed via qRT-PCR (Fig. 1d). Thereafter, lncCTSLP8 was overexpressed in ovarian cancer cell lines with relatively low lnc-CTSLP8 expression (SKOV3 and OVCA420) and knocked out in the ovarian cancer cell line with the highest lnc-CTSLP8 expression (Hey). We analyzed lnc-CTSLP8 expression in lnc-CTSLP8 overexpression/knockout $(\mathrm{OE} / \mathrm{KO})$ cell lines relative to the negative control $(\mathrm{NC})$ cell lines (cell lines transfected with control vector) and blank control cell lines (wildtype cells) (Fig. 1e). We also confirmed lnc-CTSLP8 expression in lnc-CTSLP8 overexpression/ knockout cell lines via immunofluorescence assay using an lnc-CTSL P8 RNA probe (Fig. S1A).

To explore the function of lnc-CTSLP8 in ovarian cancer, RNA sequencing and Gene Set Enrichment Analysis (GSEA) were conducted in SKOV3-CTSLP8-OE/NC cell lines, highlighting cell proliferation and metastasis as important lnc-CTSLP8-associated pathways in ovarian cancer (Fig. 2a, d). Indeed, the CCK-8 assay revealed that lnc-CTSLP8 overexpression significantly promoted SKOV3 and OVCA420 cell proliferation (Fig. 2b, Fig. S2A), while lnc-CTSLP8 knockout significantly inhibited Hey cell proliferation (Fig. 2b). Colony formation assays indicated that lnc-CTSLP8 overexpression increased the number and size of colonies in SKOV3 and OVCA420 cells (Fig. 2c, Fig. S2B, C). In contrast, lnc-CTSLP8 knockout significantly reduced the number and size of Hey cell colonies (Fig. 2c). Transwell assays revealed that cell migration and invasion were enhanced in lnc-CTSL P8 overexpression SKOV3 and OVCA420 cells (Fig. 2e,f, Fig. S2D, E), while the cell migration and invasion were attenuated after lnc-CTSLP8 knockout in Hey cells (Fig. $2 \mathrm{~g}, \mathrm{~h})$.

Lnc-CTSLP8 promotes autophagy in ovarian cancer cells GSEA suggested that autophagy was enhanced in ovarian cancer cells under lnc-CTSLP8 overexpression (Fig. 3a). TEM revealed greater numbers of enlarged autophagosomes (AU), autolysosomes (AL), and dysfunctional mitochondria (MT) following lnc-CTSLP8 knockout, whereas overexpression decreased the number of autolysosomes and dysfunctional mitochondria in ovarian cancer cells (Fig. 3b). We also found that LC3 was downregulated in lnc-CTSLP8 overexpression cells while upregulated in their knockout counterparts (Fig. S3A).

Notably, lnc-CTSLP8 overexpression/knockout had a significant effect on the expression of autophagy-specific substrate SQSTM1/p62, but not on that of molecules crucial for autophagosome formation such as autophagy-related 3 (ATG3), ATG7, and ATG12. Meanwhile, ectopic expression of lnc-CTSLP8 significantly altered the LC3-II/LC3-I ratio in ovarian cancer cells (Fig. 3c, d). mRFP-GFP-LC3 expression was then detected via immunofluorescence (Fig. 3e). Since GFP immunofluorescence could be compromised by the acidic environment within the autolysosome, we used mRFP-GFP-LC3 as an index of autophagy flux in ovarian cancer cells. Immunofluorescence assays indicated that autophagy flux was enhanced by lnc-CTSLP8 overexpression and suppressed by lnc-CTSLP8 knockout.

\section{Lnc-CTSLP8 promotes EMT in ovarian cancer cells}

GSEA suggested that the EMT was promoted in ovarian cancer cells after lnc-CTSLP8 overexpression (Fig. 4a). Immunoblotting of EMT markers E-cadherin and Ncadherin in SKOV3-CTSLP8-OE and Hey-CTSLP8-KO cell lines indicated that the EMT was indeed promoted via lnc-CTSLP8 overexpression and suppressed under lnc-CTSLP8 knockout (Fig. 4b, c). Immunofluorescence staining of E-cadherin and $\mathrm{N}$-cadherin revealed similar results (Fig. 4d). In addition, the expression of ZEB1 and 


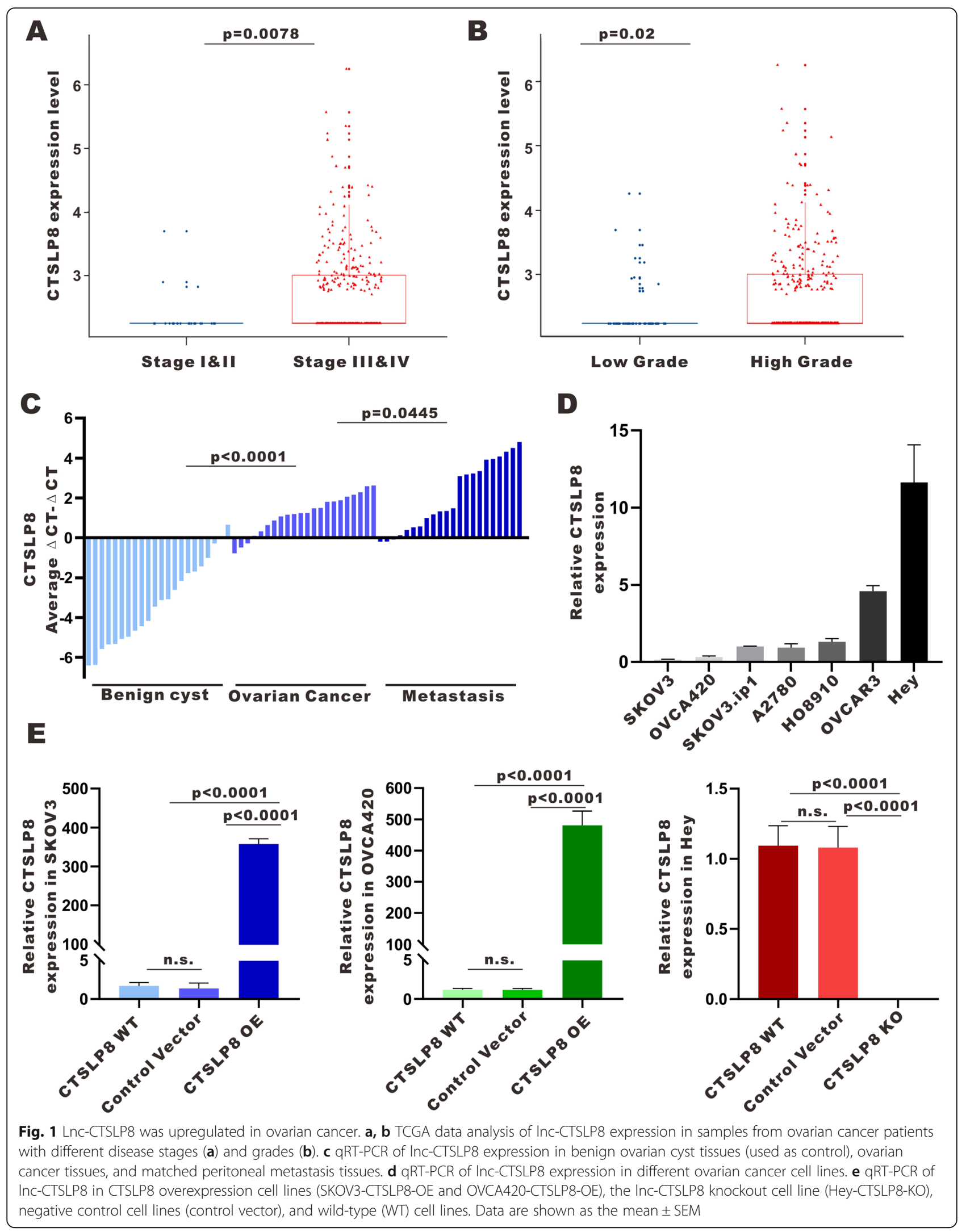



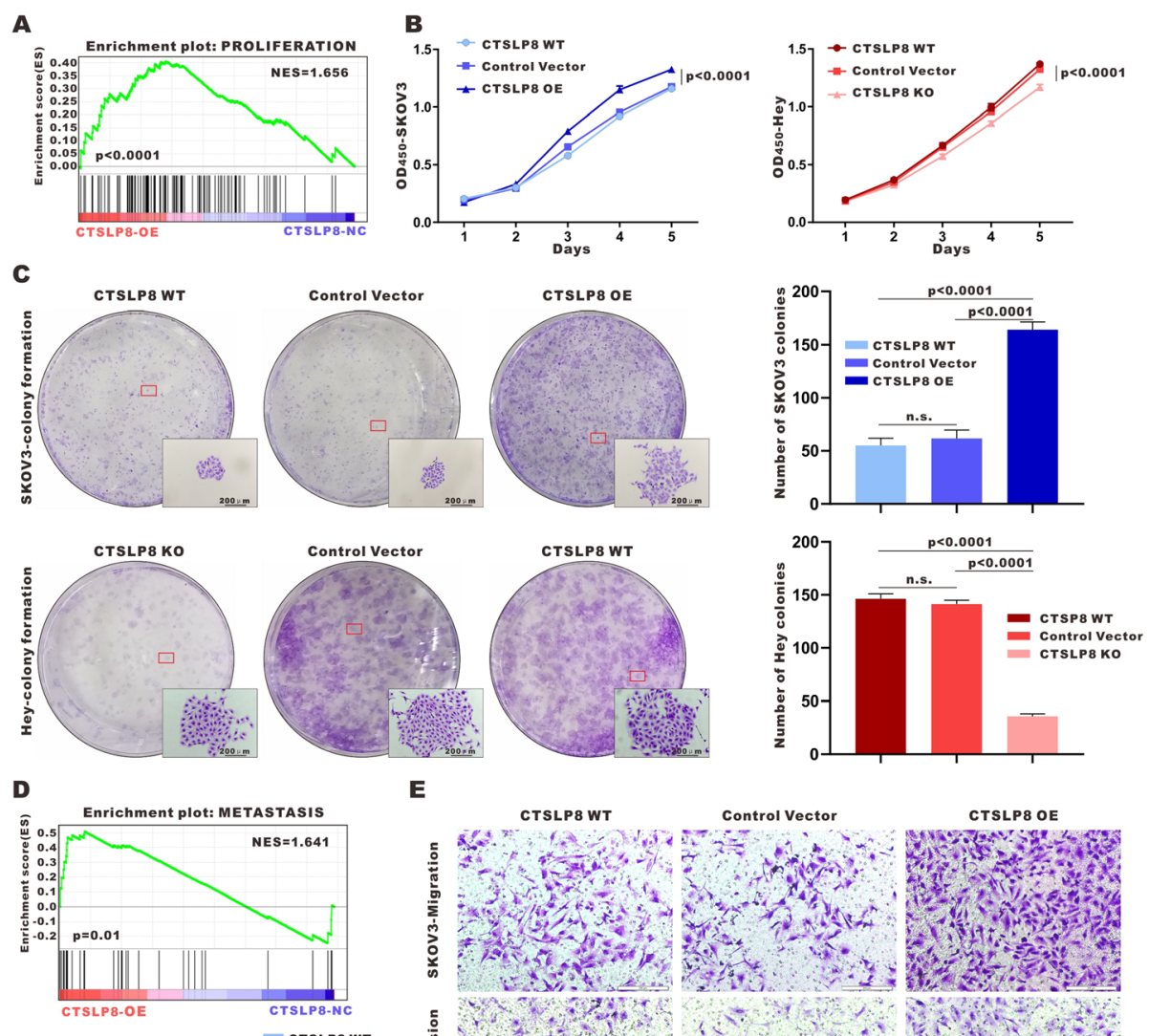

E
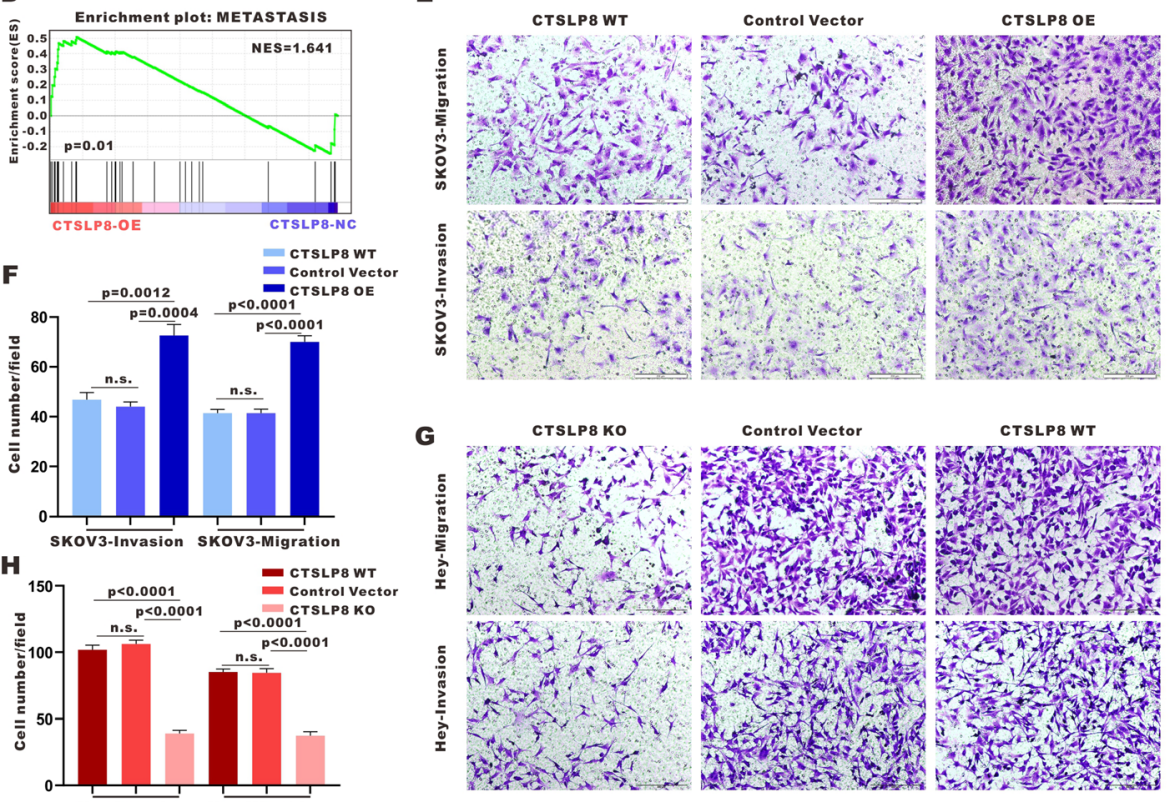

Fig. 2 Lnc-CTSLP8 promoted the proliferation and invasion of ovarian cancer cells. a and d GSEA analysis of SKOV3-CTSLP8-OE and SKOV3-CTSL P8-NC (control vector). The signature was defined by genes with significant expression changes. b Cell viability assay and (c) colony formation assay in different cell lines. $\mathbf{e - g}$ ) Invasion and migration assays in different cell lines

Snail, key regulators of the EMT, was detected via immunofluorescence staining (Fig. S3B). Overexpression of lnc-CTSLP8 upregulated while lnc-CTSLP8 knockout suppressed the expression of both regulators.

\section{Lnc-CTSLP8 promotes the tumorigenesis and metastasis of ovarian cancer in vivo}

To validate the function of lnc-CTSLP8 in vivo, we established an orthotopic ovarian cancer mouse model. As shown in Fig. 5a and d, lnc-CTSLP8 overexpression promoted the growth of SKOV3 cell-derived tumors, while lnc-CTSLP8 depletion led to decreased growth of Hey cell-derived tumors. Lnc-CTSLP8 overexpression also increased the volume and weight of SKOV3 cellderived tumors, while lnc-CTSLP8 knockout had the opposite effect in Hey cell-derived tumors (Fig. 5b, e). Moreover, lnc-CTSLP8 overexpression led to reduced overall survival in tumor-bearing mice, whereas mice with Hey-CTSLP8-KO tumors had longer overall survival than their Hey-CTSLP8-NC and Hey-CTSLP8-WT 


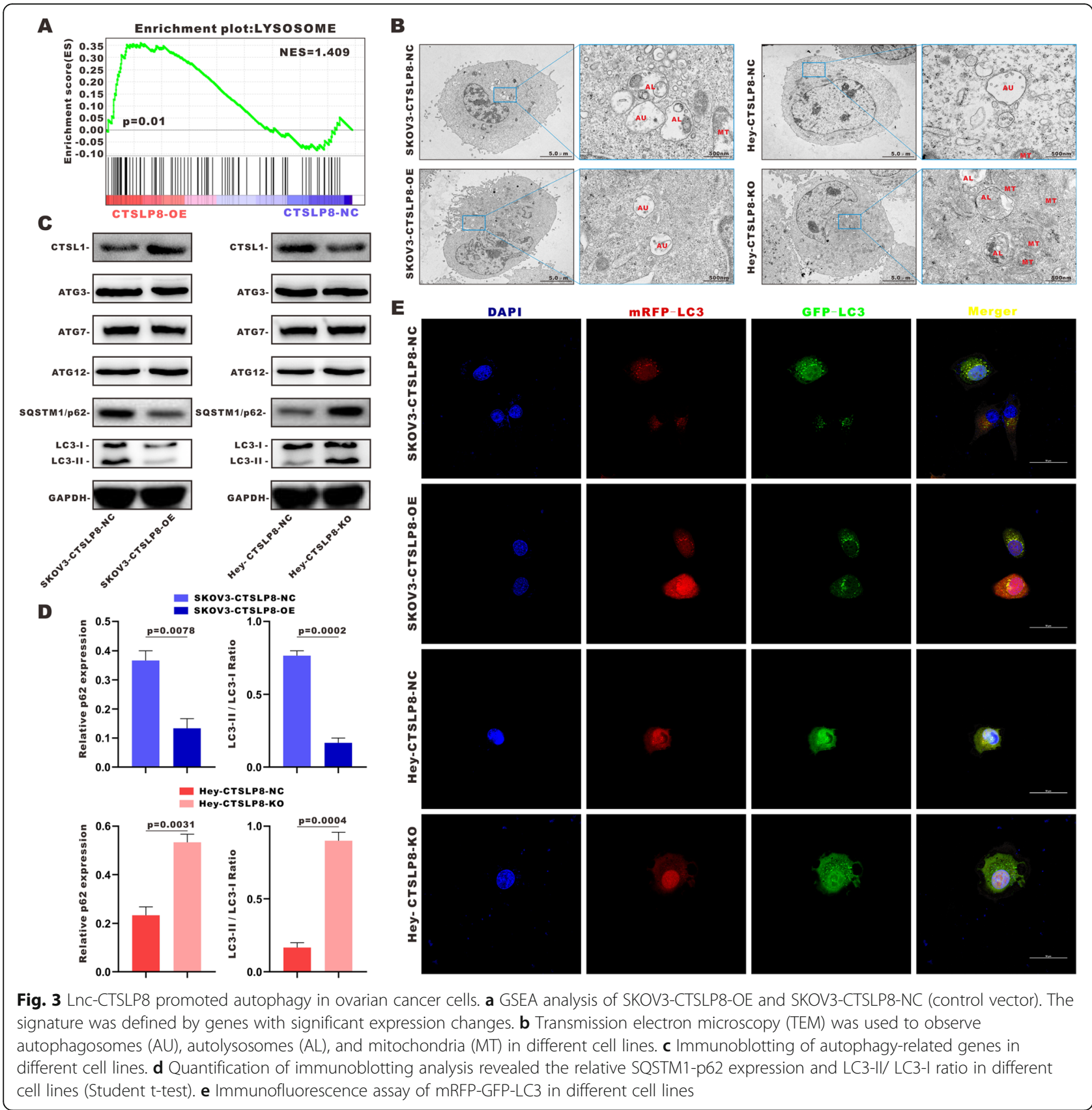

counterparts (Fig. 5c, f). Metastasis tissues in the right ovary, intestine, and peritoneum of tumor-bearing mice were harvested for HE staining (Fig. 5g). SKOV3-CTSL P8-OE-inoculated mice developed more metastases, while less metastases were observed in Hey-CTSLP8-KO mouse tumor models (Fig. 5h).

An orthotopic ovarian cancer model was generated using OVCA420-CTSLP8-OE and associated control cell lines. In agreement with previous results, lnc-CTSLP overexpression promoted OVCA420 tumor growth, volume, weight, and metastases in mice. Further, OVCA420-CTSLP8-OE-inoculated mice had significantly reduced overall survival compared to control groups (Fig. S2F-J).

The overexpression/knockout of lnc-CTSLP8 in mouse orthotopic ovarian tumor tissues was confirmed via immunofluorescence using the lnc-CTSLP8 probe (Fig. S4A). The expression of LC3, SQSTM1/p62, Ecadherin, and $\mathrm{N}$-cadherin was detected via immunofluorescence assays (Fig. S4B, C). Reduced LC3 and SQSTM1/p62 were observed in SKOV3-CTSLP8-OE ovarian tumor tissues. Further, lnc-CTSLP8 overexpression upregulated N-cadherin, indicating that lnc-CTSL P8 might promote ovarian cancer autophagy and EMT 


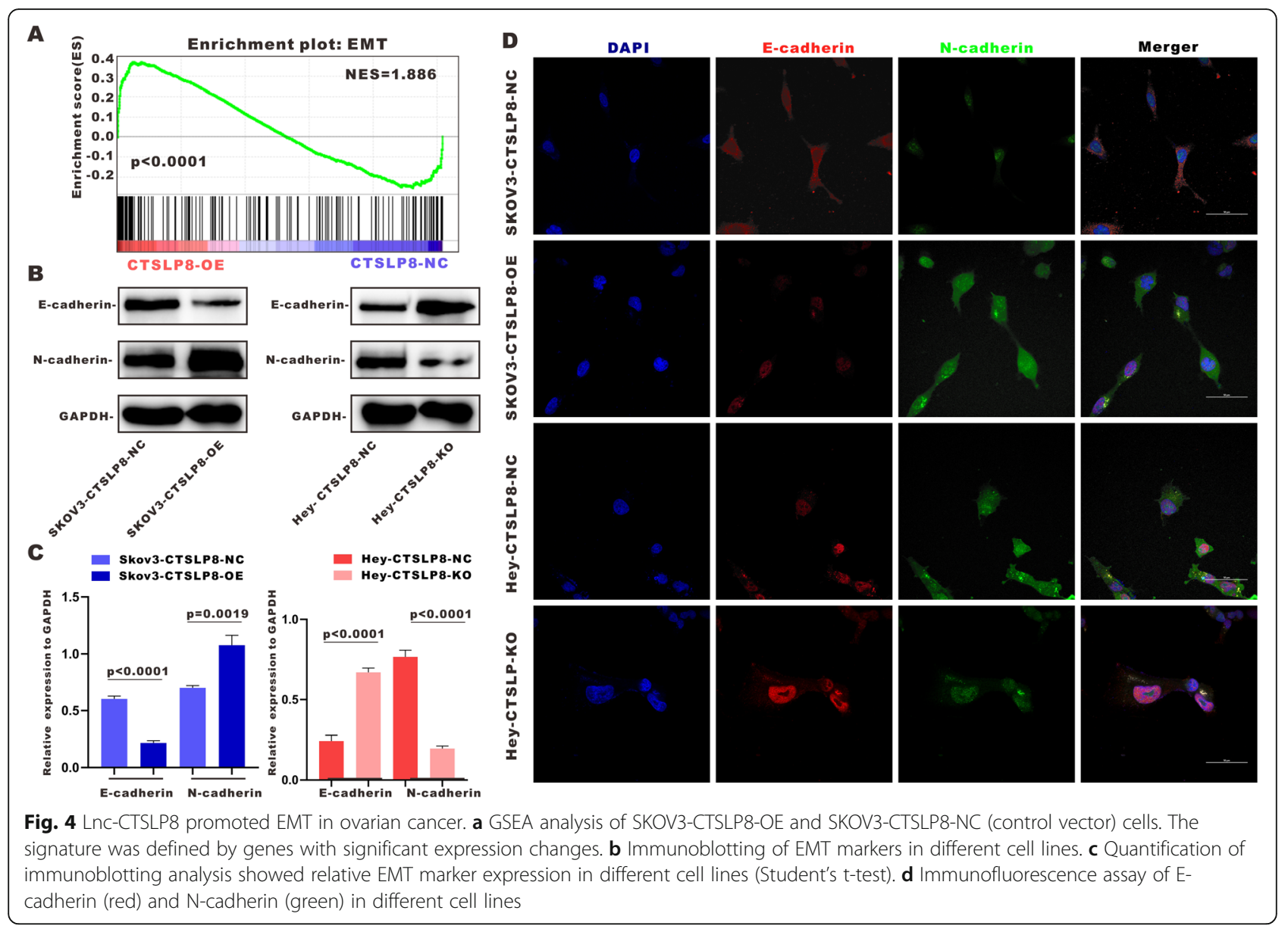

in vivo. In contrast, we observed suppressed autophagy and EMT in the ovarian tumor tissues of Hey-CTSLP8$\mathrm{KO}$ mice.

\section{Lnc-CTSLP8 acts as a sponge for miR-199a-5p in ovarian cancer}

Using qRT-PCR (Fig. 6a), we found that CTSL1 mRNA expression was also significantly elevated in ovarian cancer tumor tissues $(n=22)$ relative to benign ovarian cyst tissues $(n=22)$ and highest in ovarian cancer peritoneal metastasis tissues $(n=22)$. Furthermore, CTSL1 expression was positively correlated with lnc-CTSLP8 expression in different tissues (Fig. 6b). The same positive correlation was found via GEO ovarian cancer data analysis (Fig. 6c).

We screened miRNA with potential binding sites for both lnc-CTSLP8 and CTSL1-3'UTR. miR-199a-5p and miR-3667-3p were identified using the TargetScan public database (Fig. 6d). To validate the direct binding of miR-199a-5p with both lnc-CTSLP8 and CTSL1, biotinlabeled miR-199a-5p and its mutant mimics were designed to pull down lnc-CTSLP8 and CTSL1 in SKOV3 cells overexpressing lnc-CTSLP8. We found obvious enrichment of lnc-CTSLP8 and CTSL1 for wild-type miR- 199a-5p compared with mutant mimics (Fig. 6e). An anti-AGO2 RIP assay was then performed, wherein lncCTSLP8, CTSL1, and miR-199a-5p were enriched with AGO2 (Fig. 6f).

Additionally, we tested the predicted shared miRNAs in a luciferase reporter assay and found that miR-199a-5p significantly repressed lnc-CTSLP8 and CTSL1-3'UTR luciferase activity. The mutation of the predicted miRNA binding sites in lnc-CTSLP8 and CTSL1-3'UTR abrogated this effect (Fig. 6g), suggesting that the crosstalk between lnc-CTSLP8 and CTSL1 may be mediated, at least in part, by miR-199a-5p. Moreover, CTSL1 expression was upregulated after lnc-CTSLP8 overexpression and downregulated after lnc-CTSLP8 knockout (Fig. 6h).

The miR-199a-5p expression was significantly decreased in ovarian cancer peritoneal metastasis tissue (Fig. S5A). Further, miR-199a-5p expression exhibited negative correlation with CTSL1 expression (Fig. S5B). Meanwhile, miR-199a-5p expression was significantly altered by the overexpression and knockout of lnc-CTSL P8 (Fig. S5C).

These results indicated that lnc-CTSLP8 may function as a sponge for miR-199a-5p and upregulate CTSL1 as a ceRNA. 


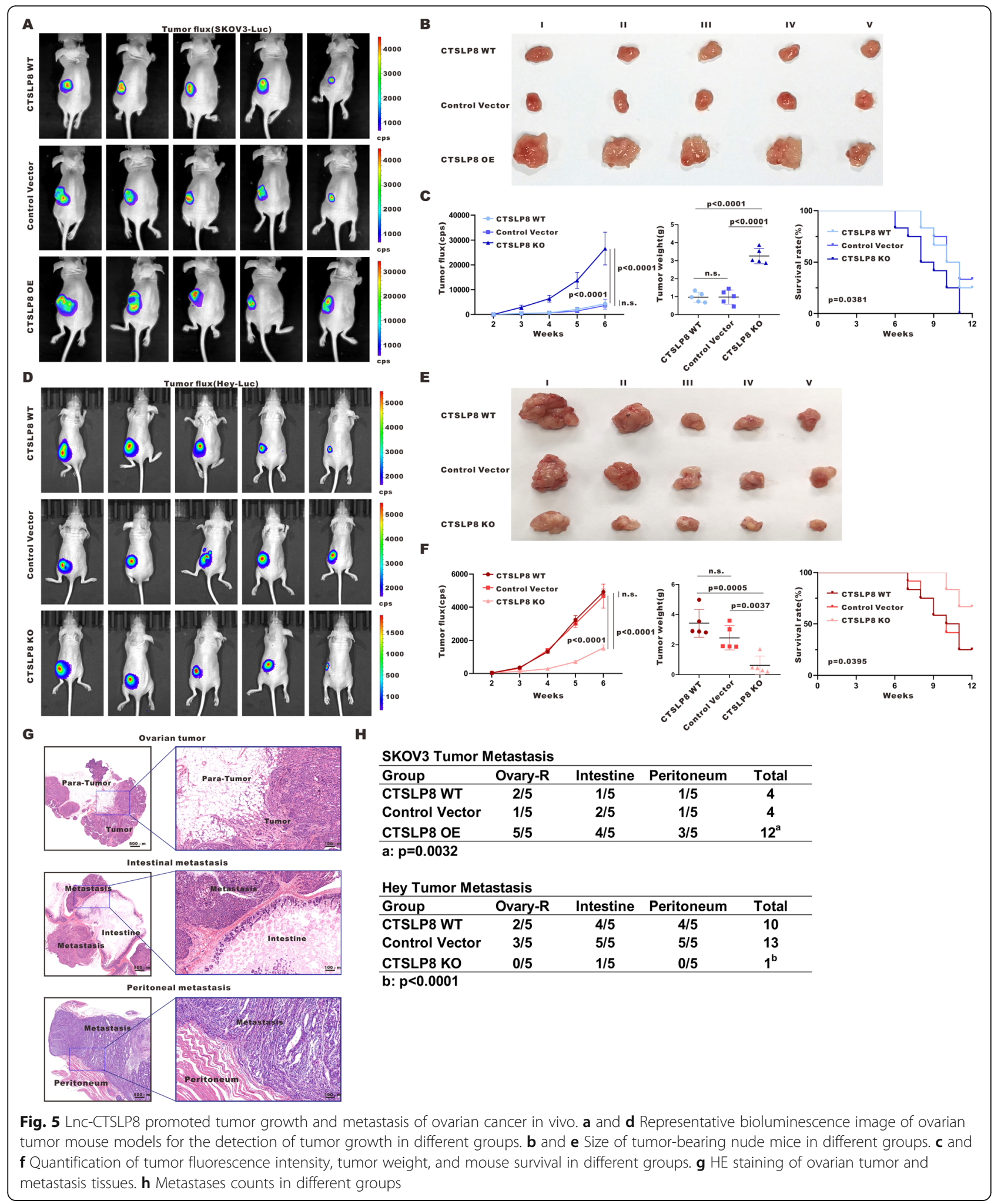

CTSL1 inhibitor reversed the oncogenic effects of IncCTSLP8 in vitro and in vivo

We isolated single cells from para-tumor tissues (used as normal controls), ovarian cancer tissues, and peritoneal metastasis tissues from three high-grade serous ovarian cancer patients. Since we barely found CTSL1-positive cells $\left(\mathrm{CTSL}^{+}{ }^{+}\right.$cells) in para-tumor tissues, the cell markers expression of $\mathrm{CTSL}^{+}$cells were only analyzed 


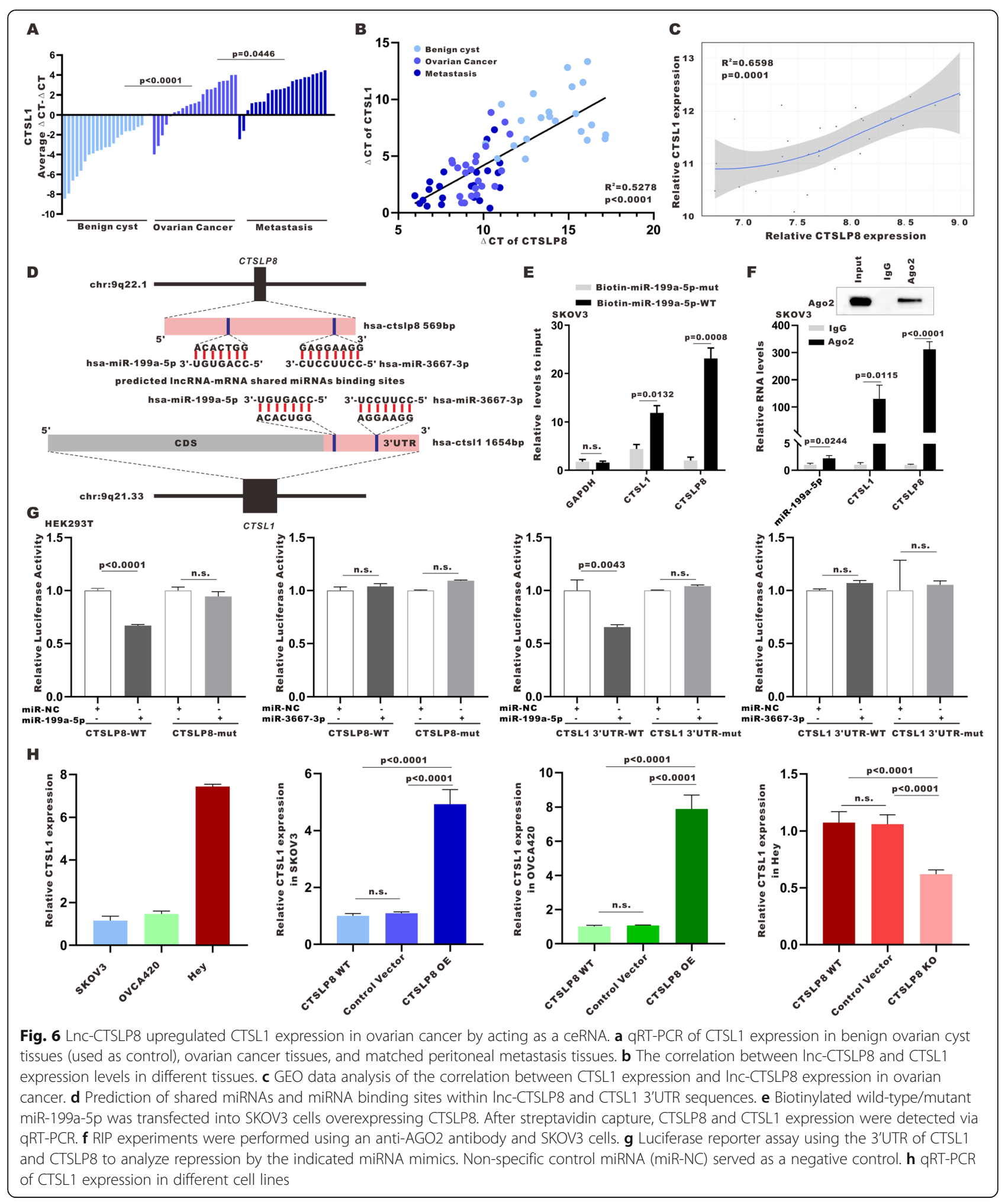

in ovarian cancer and peritoneal metastasis tissues. High EPCAM expression was observed in $\mathrm{CTSL}^{+}$cells (Fig. 7a), indicating that CTSL1 within the ovarian cancer microenvironment was most likely derived from tumor cells (EPCAM ${ }^{+}$cells). The expression of monocyte/macrophage marker CD14 and $\mathrm{T}$ cell marker CD4 was detected in CTSL $^{+}$cells (Fig. 7a). The number of $\mathrm{CTSL}^{+} \mathrm{EPCAM}^{+}$cells and the 


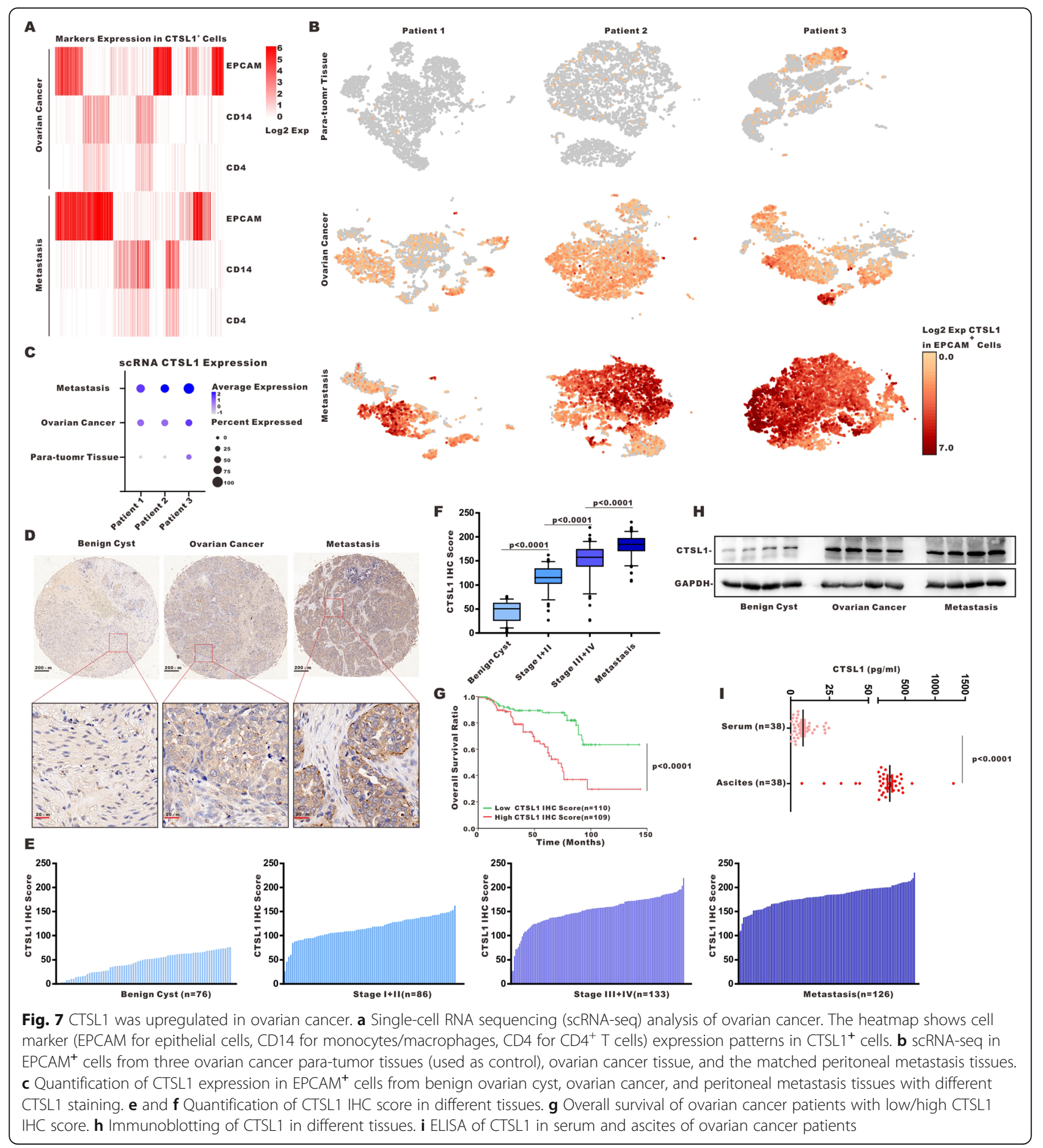

expression level of CTSL1 in $\mathrm{EPCAM}^{+}$cells were both higher in ovarian cancer tissues than in paratumor tissues. Further, peritoneal metastasis tissues had the highest number of CTSL1 ${ }^{+} \mathrm{EPCAM}^{+}$cells as well as the highest expression level of CTSL1 in these cells (Fig. 7b, c). Similar CTSL1 expression patterns were observed in cells from different tissues (Fig. S6A).
Next, we evaluated CTSL1 protein expression in ovarian cancer tissue. The CTSL1 IHC score of ovarian cancer tissue was significantly higher than that of benign ovarian cyst tissues (Fig. 7d). Moreover, the CTSL1 IHC score increased with disease progression (Fig. 7e, f), and high CTSL1 expression was associated with poor overall survival in ovarian cancer patients (Fig. $7 \mathrm{~g}$ ). The upregulated CTSL1 expression in ovarian cancer and peritoneal 


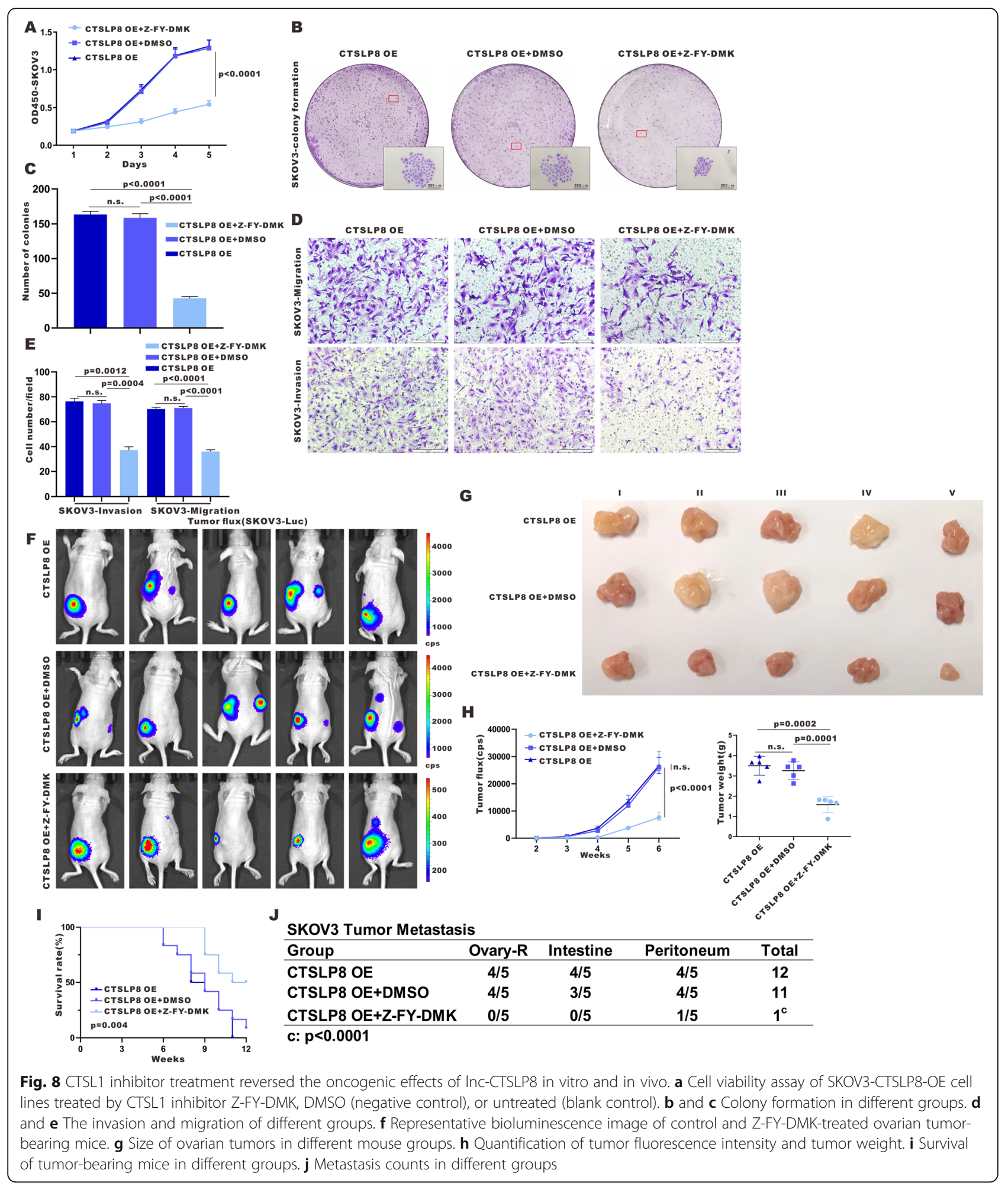

metastasis tissues was further confirmed via western blot analysis (Fig. 7h). ELISA revealed that soluble CTSL1 was significantly higher in ascites compared to serum obtained from the same cohort of ovarian cancer patients (Fig. 7i).
Next, we inhibited CTSL1 activity in lnc-CTSLP8overexpressing ovarian cancer cells using Z-FY-DMK, a selective CTSL1 inhibitor. Ectopic expression of lncCTSLP8 enhanced the proliferation, migration, and invasion of ovarian cancer cells, but these effects were 
abrogated by Z-FY-DMK (Fig. 8a-e). Similarly, lnc-CTSL P8 overexpression enhanced tumor formation and tumor weight in vivo, yet these effects were also suppressed in mice treated with Z-FY-DMK (Fig. 8f-g). Further, Z-FYDMK improved the overall survival of and reduced tumor metastasis in SKOV3-CTSLP8-OE tumor-bearing mice (Fig. 8i,j).

Immunofluorescence assay using the lnc-CTSLP8 probe confirmed that lnc-CTSLP8 overexpression was not affected by Z-FY-DMK treatment (Fig. S7A). The expression of LC3, SQSTM1/p62, E-cadherin, and Ncadherin was then detected via immunofluorescence (Fig. S7B, C). Increased LC3 and SQSTM1/p62 levels were observed in Z-FY-DMK-treated SKOV3-CTSLP8$\mathrm{OE}$ ovarian tumor tissues. Further, the EMT process was inhibited by Z-FY-DMK.

\section{CTSL1 inhibitor and miR-199a-5p overexpression abrogated Inc-CTSLP8-promoted EMT and autophagy in ovarian cancer}

The number of enlarged autophagosomes (AU), autolysosomes (AL), and dysfunctional mitochondria (MT) increased in SKOV3-CTSLP8-OE cells treated with Z-FYDMK, but not in their DMSO-treated counterparts (Fig. 9a). Z-FY-DMK treatment elevated SQSTM1/p62 expression and the LC3-II/LC3-I ratio in SKOV3-CTSL P8-OE cells (Fig. 9c, d), indicating that the promotion of autophagy by lnc-CTSLP8 overexpression was reversed by the CTSL1 inhibitor. The blockade of autophagy flux by Z-FY-DMK was then confirmed via mRFP-GFP-LC3 immunofluorescence (Fig. 9h).

As an CTSL1-targeting miRNA, miR-199a-5p mediates ceRNA regulation between lnc-CTSLP8 and CTSL1. Therefore, miR-199a-5p was transfected into SKOV3CTSLP8-OE cells to confirm its regulatory role. TEM analyses revealed that miR-199a-5p increased the number of enlarged AU, AL, and dysfunctional mitochondria MT in SKOV3-CTSLP8-OE cells relative to miR-NC (Fig. 9b). Immunoblotting analyses indicated that CTSL1 expression was decreased by miR-199a-5p. The SQST M1/p62 expression and LC3-II/LC3-I ratio were elevated in SKOV3-CTSLP8-OE cells (Fig. 9e, f), suggesting that the promotion of autophagy by lnc-CTSLP8 overexpression should be reversed by miR-199a-5p. The blockade of autophagy flux by miR-199a-5p was then confirmed via mRFP-GFP-LC3 immunofluorescence detection (Fig. 9g). Immunofluorescence assays of LC3 in SKOV3CTSLP8-OE cells treated with Z-FY-DMK or miR-199a$5 p$ were conducted, revealing that LC3 expression increased after Z-FY-DMK or miR-199a-5p treatment (Fig. S8A, D).

Furthermore, the EMT promoted by lnc-CTSLP8 overexpression was also abrogated in cells treated with Z-FY-DMK or miR-199a-5p, since the expression of E- cadherin was significantly increased, while that of $\mathrm{N}$ cadherin was significantly reduced, as determined via immunoblotting (Fig. 9c, f). Immunofluorescence assays of E-cadherin and $\mathrm{N}$-cadherin confirmed these results (Fig. S8C, F). In addition, the expression of ZEB1 and Snail, key regulators of the EMT, was detected by immunofluorescence staining (Fig. S8B, E), showing that ZFY-DMK and miR-199a-5p reduced the expression of both regulators in SKOV3-CTSLP8-OE cells.

\section{Discussion}

Pseudogenes are involved in human malignancies through their function as ceRNAs for their cognate genes due to sharing the same miRNA response elements [23]. Whether pseudogenes function as tumor suppressors or oncogenes depends on their proteincoding gene counterparts. For instance, pseudogene ceRNA networks of BRAFP1-BRAF [17] and KRASP1KRAS [24] were shown to promote tumorigenesis. In this study, we investigated the role of pseudogenes in ovarian cancer, with a particular focus on CTSL pseudogene 8 , as qRT-PCR confirmed its upregulation in metastatic ovarian cancer tissues. Further, public database analysis also revealed lnc-CTSLP8 upregulation in advanced-stage or high-grade ovarian cancer patients. Through overexpression/knockout of lnc-CTSLP8 in ovarian cancer cells in vitro and in vivo, we established lnc-CTSLP8 as a potent oncogene, which acts by enhancing the autophagy and EMT in ovarian cancer cells.

Mechanistically, lnc-CTSLP8 contributed to the progression of ovarian cancer by acting as a ceRNA to its cognate gene, CTSL1. Following previous models of ceRNA interactions $[25,26]$, we confirmed the regulation between lnc-CTSLP8 and CTSL1 via miRNA binding prediction, RIP, and target site mutation analyses.

CTSL1, a family member of cysteine cathepsins, is a crucial lysosomal enzyme. Tumor cell-derived CTSL1 was reported to have a tumor-promoting function, as the CTSL1 knockout phenotype in mice could not be rescued via transplantation of wild-type donor bone marrow [27]. In this study, we confirmed that ovarian cancer cells were the major source of CTSL1, and soluble CTSL1 was enriched in malignant ascites fluid. CTSL1 deficiency could lead to defects in autophagy, characterized by large dysmorphic vesicles and the accumulation of mitochondria within the cytoplasm [28]. Moreover, defects in autophagy could also be characterized by an increased LC3-II/LC3-I ratio and the accumulation of the autophagy substrate SQSTM1/p62 [29]. We observed these autophagy defects in lnc-CTSLP8 knockout ovarian cancer cells. Secreted CTSL1 was reported to promote cancer cell invasion and EMT by cleaving cell adhesion molecule E-cadherin [19]. As expected, we detected increased $\mathrm{N}$-cadherin and reduced 

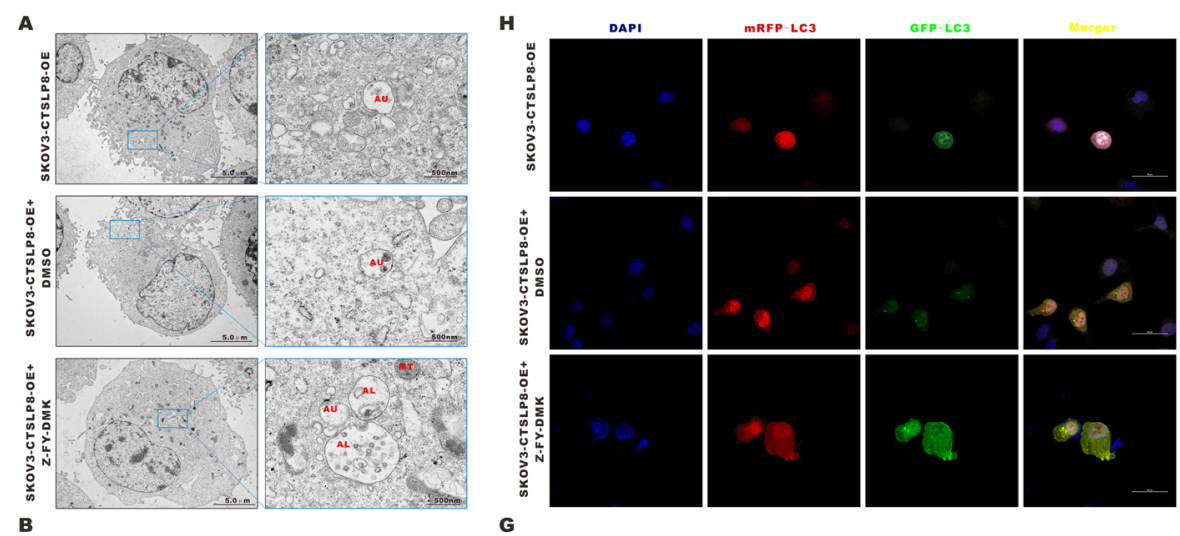

G
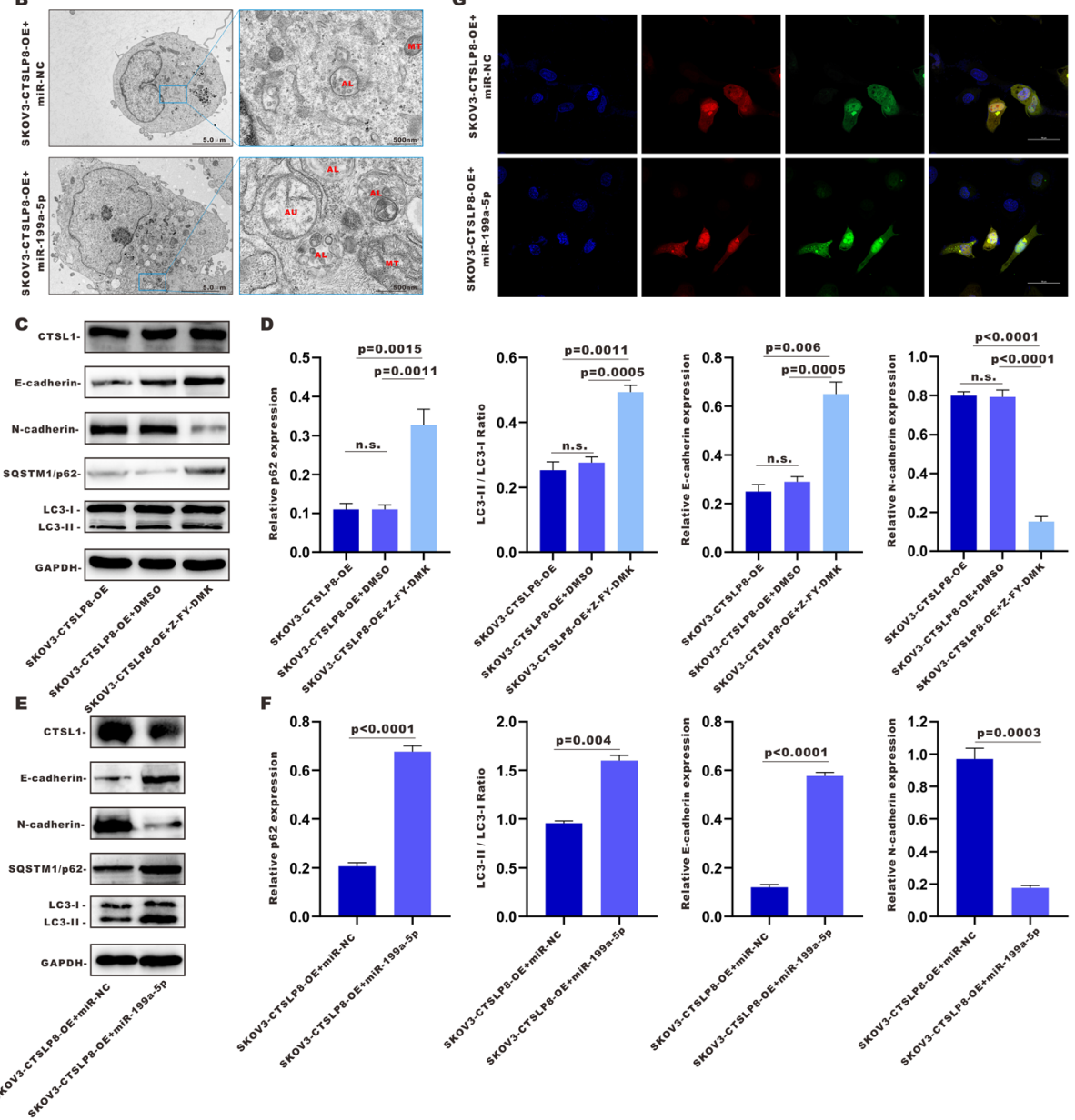

$\mathbf{F}$
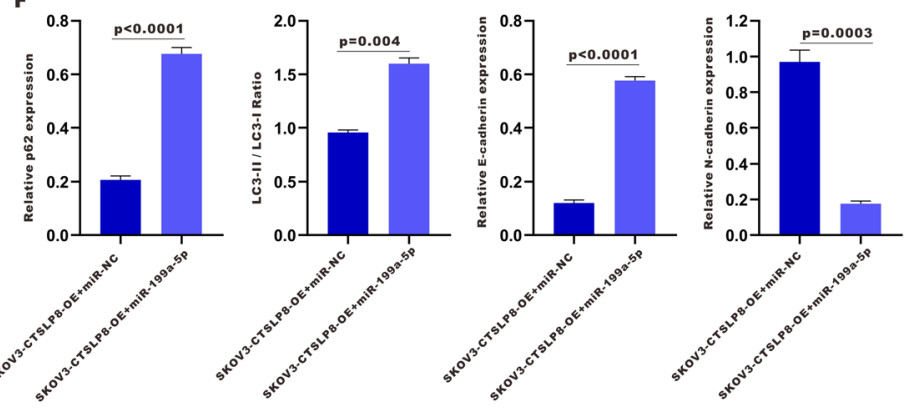

Fig. 9 CTSL1 inhibitor and miR-199a-5p abrogated the Inc-CTSLP8-mediated promotion of EMT and autophagy in ovarian cancer. a TEM of the $A U, A L$, and MT in SKOV3-CTSLP8-OE cells treated with CTSL1 inhibitor Z-FY-DMK, DMSO (negative control), or untreated (blank control). b TEM of the AU, AL, and MT in SKOV3-CTSLP8-OE cells treated with miR-199a-5p or miR-NC (negative control). c and e Immunoblotting of autophagyrelated genes and EMT markers in different cell lines. $\mathbf{d}$ and $\mathbf{f}$ Quantification of relative p62 expression, LC3-II/ LC3-I ratio, and EMT marker expression in different cell lines. $\mathbf{h}$ and $\mathbf{f}$ Immunofluorescence assay of mRFP-GFP-LC3 in different cell lines

E-cadherin in lnc-CTSLP8 OE ovarian expression. Surprisingly, EMT-related transcription factors ZEB1 and Snail were also regulated by lnc-CTSLP8, and further studies are needed to elucidate the underlying mechanisms.
CTSL1 is dysregulated in various human diseases and represents a promising drug target [30, 31]. In this study, CTSL1 inhibition abrogated the oncogenic effects of lncCTSLP8. In particular, the enhancement of autophagy and EMT observed in lnc-CTSLP8 OE cancer cells was 
reversed by treatment with a selective CTSL1 inhibitor, indicating that the function of lnc-CTSLP8 depended on CTSL1 activity. Whether the oncogenic effects of lncCTSLP8 involve other ceRNA targets remains to be addressed in further research.

\section{Conclusion}

In summary, lnc-CTSLP8 upregulates CTSL1 by acting as a decoy against their shared miRNA, miR-199a-5p. Consequently, the pseudogene-gene functional network promotes ovarian cancer metastasis via the induction of EMT and autophagy in cancer cells. Moreover, the oncogenic effects of lnc-CTSLP8 can be abrogated by CTSL1 selective inhibitor treatment. Collectively, our study reveals a novel ceRNA mechanism and identifies lnc-CTSLP8 as a potential therapeutic target for metastatic ovarian cancer.

\section{Abbreviations}

IncRNA: Long non-coding RNA; CTSLP8: Cysteine cathepsin L pseudogene 8; CTSL1: Cysteine cathepsin L 1; ceRNA: Competing endogenous RNA: PTEN: Phosphatase and tensin homolog; BRAF: B-Raf proto-oncogene, serine/ threonine kinase; KRAS: KRAS proto-oncogene, GTPase; TCGA: The Cancer Genome Atlas; GEO: Gene Expression Omnibus; ZEB1: Zinc finger E-box binding homeobox 1; LC3: Microtubule associated protein 1 light chain 3 alpha; EMT: Epithelial-mesenchymal transition; EPCAM: Epithelial cell adhesion molecule; WT: Wild type; OE: Overexpression; KO: Knock out; NC: Negative control

\section{Supplementary Information}

The online version contains supplementary material available at https://doi. org/10.1186/s13046-021-01957-z.

Additional file 1: Supplementary Table 1. The clinicopathological characteristics and overall survival in ovarian cancer patients with different CTSL1 expression.

Additional file 2: Supplementary Table 2. sgRNA sequence. Additional file 3: Supplementary Table 3. Primer sequence. Additional file 4: Supplementary Table 4. Primary antibodies.

Additional file 5: Figure S1. CTSLP8 overexpression and knockout cell lines were constructed for further study. (A) Immunofluorescence assay using an RNA probe designed for CTSLP8 (Red) in CTSLP8 overexpression and knockout cell lines. (B) sgRNAs designed for CTSLP8 knockout. (C) Structure of the two-gRNA-expressing plasmid. (D) Sanger DNA sequencing of knockout cell line.

Additional file 6: Figure S2. CTSLP8 overexpression promoted ovarian cancer progression in vitro and in vivo. (A) Viability assay of OVCA420CTSLP8-OE cells, OVCA420 cells transfected with a control vector (negative control), and wild-type OVCA420 (blank control). Significance was calculated via the two-way ANOVA test. (B and C) The colony formation of different groups (Student's t-test). ( $\mathrm{D}$ and $\mathrm{E}$ ) The invasion and migration of different groups (Student's t-test). (F) Representative bioluminescence image of control and CTSLP8-OE OVCA420 ovarian tumor-bearing mice. (G) Size of ovarian tumors in different mouse groups. (H) Quantification of tumor fluorescence intensity (two-way ANOVA test) and tumor weight (Student's t-test). (I) Tumor-bearing mouse survival in different groups (Kaplan-Meier survival analysis). (J) Metastasis counts in different groups (chi-square test).

Additional file 7: Figure S3. LnC-CTSLP8 regulated LC3 and EMTrelated transcription factors in ovarian cancer cells. (A) Immunofluorescence assay of LC3 (red) in different cell lines. (B) Immunofluorescence assay of EMT-related transcription factors ZEB1 (red) and Snail (green) in different cell lines.

Additional file 8: Figure S4. LnC-CTSLP8 promoted EMT and autophagy in ovarian cancer in vivo. (A) Immunofluorescence assay using an RNA probe design for CTSLP8 (red) in ovarian cancer tissues of tumorbearing mice. (B) Immunofluorescence assay for LC3 (red) and p62 (green) in ovarian cancer tissues of tumor-bearing mice.

Additional file 9: Figure S5. miR-199a-5p was negatively correlated with CTSL1 expression in ovarian cancer. (A) qRT-PCR of relative miR199a-5p expression in benign ovarian cyst tissues (used as control), ovarian cancer tissues, and matched peritoneal metastasis tissues (Student's ttest). (B) Correlation analysis between miR-199a-5p and CTSL1 expression levels in different tissues (linear regression analysis). (C) qRT-PCR of relative CTSL1 expression in different cell lines (Student's t-test).

Additional file 10: Figure S6. CTSL1 expression was significantly elevated in ovarian cancer tissues and metastasis tissues. (A) scRNA-seq in all cells from three ovarian cancer para-tumor tissues (used as control), ovarian cancer tissues, and the matched peritoneal metastasis tissues.

Additional file 11: Figure S7. CTSL1 inhibition reversed the CTSLP8mediated promotion of EMT and autophagy in ovarian cancer in vivo. (A) Immunofluorescence assay using an RNA probe against CTSLP8 (red) in ovarian cancer tissues of tumor-bearing mice. (B) Immunofluorescence assay for LC3 (red) and p62 (green) in ovarian cancer tissues of tumorbearing mice.

Additional file 12: Figure S8. CTSL1 inhibition and miR-199a-5p reversed the CTSLP8-mediated promotion of EMT and autophagy in ovarian cancer cells. (A and D) Immunofluorescence assay of LC3 (red) in different cell lines. (B and E) Immunofluorescence assay of EMT-related transcription factors ZEB1 (red) and Snail (green) in different cell lines. (C and F) Immunoblotting of EMT markers in different cell lines.

\section{Acknowledgements}

The RNA expression and ovarian cancer patient clinical information were obtained from the TCGA and GEO Research Network.

\section{Authors' contributions}

Xipeng Wang, Ziliang Wang, and Xinjing Wang conceived the study. Xinjing Wang and Xiaoduan Li acquired patient samples and performed the experiments and data analyses. Huizhen Sun, Feikai Lin, and Yingying Lin analyzed and interpreted the data. Xipeng Wang and Xinjing Wang wrote and revised the manuscript. All authors read and approved the final manuscript.

\section{Funding}

National Natural Science Foundation of China $(81930064,81874103$ to Xipeng Wang, 81602280 to Xinjing Wang, 81872117 to Ziliang Wang).

\section{Availability of data and materials}

All data used or analyzed during this study are included in this article and are available from the corresponding author upon reasonable request.

\section{Declarations}

\section{Ethics approval and consent to participate}

The study protocols were approved by the Ethics Committee of Xinhua Hospital, Shanghai Jiaotong University School of Medicine. Informed consent was obtained from all participants included in this study according to ethical committee regulations.

Consent for publication

All authors agree with the content of the manuscript.

\section{Competing interests}

The authors declare that they have no competing interests.

\section{Author details}

${ }^{1}$ Department of Gynecology and Obstetrics, XinHua Hospital, Shanghai JiaoTong University School of Medicine, 1665 Kongjiang Rd, Yangpu District, Shanghai 200092, China. 'Department of Gynecology, Shanghai First 
Maternity and Infant Hospital, Tongji University School of Medicine, Shanghai, China. ${ }^{3}$ Department of Neurosurgery, Renji Hospital, Shanghai JiaoTong University School of Medicine, Shanghai, China.

Received: 18 January 2021 Accepted: 20 April 2021

Published online: 01 May 2021

\section{References}

1. Siegel RL, Miller KD, Jemal A. Cancer statistics, 2019. CA Cancer J Clin. 2019; 69(1):7-34. https://doi.org/10.3322/caac.21551.

2. Chen W, Zheng R, Baade PD, Zhang S, Zeng H, Bray F, et al. Cancer statistics in China, 2015. CA Cancer J Clin. 2016;66(2):115-32. https://doi.org/10.3322/ caac. 21338 .

3. Henderson JT, Webber EM, Sawaya GF. Screening for ovarian cancer: updated evidence report and systematic review for the US preventive services task force. JAMA. 2018;319(6):595-606. https://doi.org/10.1001/ja ma.2017.21421.

4. Coleman RL, Monk BJ, Sood AK, Herzog TJ. Latest research and treatment of advanced-stage epithelial ovarian cancer. Nat Rev Clin Oncol. 2013;10(4) 211-24. https://doi.org/10.1038/nrclinonc.2013.5.

5. Wang Z, Liu H, Xu C. Cellular senescence in the treatment of ovarian cancer. Int J Gynecol Cancer. 2018;28(5):895-902. https://doi.org/10.1097/IGC. 0000000000001257.

6. Gutschner T, Diederichs S. The hallmarks of cancer: a long non-coding RNA point of view. RNA Biol. 2012;9(6):703-19. https://doi.org/10.4161/rna.20481.

7. Mitra R, Chen X, Greenawalt EJ, Maulik U, Jiang W, Zhao Z, et al. Decoding critical long non-coding RNA in ovarian cancer epithelial-to-mesenchymal transition. Nat Commun. 2017;8(1):1604. https://doi.org/10.1038/s41467-01701781-0.

8. Wang X, Dai Z, Wu X, Wang K, Wang X. Distinct RNA transcriptome patterns are potentially associated with angiogenesis in Tie2-expressing monocytes. Gene. 2016;580(1):1-7. https://doi.org/10.1016/j.gene.2015.12.065.

9. Zhou J, Li X, Wu X, Zhang T, Zhu Q, Wang X, et al. Exosomes released from tumor-associated macrophages transfer miRNAs that induce a Treg/Th17 cell imbalance in epithelial ovarian cancer. Cancer Immunol Res. 2018;6(12): 1578-92. https://doi.org/10.1158/2326-6066.CIR-17-0479.

10. Salmena L, Poliseno L, Tay Y, Kats L, Pandolfi PP. A ceRNA hypothesis: the Rosetta stone of a hidden RNA language? Cell. 2011;146(3):353-8. https:// doi.org/10.1016/j.cell.2011.07.014.

11. Wee LM, Flores-Jasso CF, Salomon WE, Zamore PD. Argonaute divides its RNA guide into domains with distinct functions and RNA-binding properties. Cell. 2012;151(5):1055-67. https://doi.org/10.1016/j.cell.2012.10.036.

12. Zheng $Z Q$, Li ZX, Zhou GQ, Lin L, Zhang LL, Lv JW, et al. Long noncoding RNA FAM225A promotes nasopharyngeal carcinoma tumorigenesis and metastasis by acting as ceRNA to sponge miR-590-3p/miR-1275 and upregulate ITGB3. Cancer Res. 2019;79(18):4612-26. https://doi.org/10.1158/ 0008-5472.CAN-19-0799.

13. Credendino SC, Bellone ML, Lewin N, Amendola E, Sanges R, Basu S, et al. A ceRNA circuitry involving the long noncoding RNA KIhl14-AS, Pax8, and Bcl2 drives thyroid carcinogenesis. Cancer Res. 2019;79(22):5746-57. https://doi. org/10.1158/0008-5472.CAN-19-0039.

14. Poliseno L. Pseudogenes: newly discovered players in human cancer. Sc Signal. 2012;5(242):re5

15. Chiefari E, liritano S, Paonessa F, le Pera I, Arcidiacono B, Filocamo M, et al. Pseudogene-mediated posttranscriptional silencing of HMGA1 can result in insulin resistance and type 2 diabetes. Nat Commun. 2010;1(1):40. https:// doi.org/10.1038/ncomms1040.

16. Johnsson P, Ackley A, Vidarsdottir L, Lui WO, Corcoran M, Grandér D, et al. A pseudogene long-noncoding-RNA network regulates PTEN transcription and translation in human cells. Nat Struct Mol Biol. 2013;20(4):440-6. https:// doi.org/10.1038/nsmb.2516.

17. Karreth FA, Reschke M, Ruocco A, Ng C, Chapuy B, Léopold V, et al. The BRAF pseudogene functions as a competitive endogenous RNA and induces lymphoma in vivo. Cell. 2015;161(2):319-32. https://doi.org/10.1016/ j.cell.2015.02.043

18. Dennemärker J, Lohmüller T, Müller S, Aguilar SV, Tobin DJ, Peters C, et al. Impaired turnover of autophagolysosomes in cathepsin $L$ deficiency. Biol Chem. 2010;391(8):913-22. https://doi.org/10.1515/BC.2010.097.

19. Gocheva V, Zeng W, Ke D, Klimstra D, Reinheckel T, Peters C, et al. Distinct roles for cysteine cathepsin genes in multistage tumorigenesis. Genes Dev. 2006;20(5):543-56. https://doi.org/10.1101/gad.1407406.
20. Sobotič B, Vizovišek M, Vidmar R, van Damme P, Gocheva V, Joyce JA, et al. Proteomic identification of cysteine cathepsin substrates shed from the surface of cancer cells. Mol Cell Proteomics. 2015;14(8):2213-28. https://doi. org/10.1074/mcp.M114.044628.

21. Nishida Y, Kohno K, Kawamata T, Morimitsu K, Kuwano M, Miyakawa I. Increased cathepsin L levels in serum in some patients with ovarian cancer: comparison with CA125 and CA72-4. Gynecol Oncol. 1995;56(3):357-61. https://doi.org/10.1006/gyno.1995.1063.

22. Zhang $H$, et al. Knockdown of cathepsin $L$ sensitizes ovarian cancer cells to chemotherapy. Oncol Lett. 2016;11(6):4235-9. https://doi.org/10.3892/ol.201 6.4494 .

23. Tay Y, Rinn J, Pandolfi PP. The multilayered complexity of ceRNA crosstalk and competition. Nature. 2014;505(7483):344-52. https://doi.org/10.1038/na ture12986.

24. Poliseno L, Salmena L, Zhang J, Carver B, Haveman WJ, Pandolfi PP. A coding-independent function of gene and pseudogene mRNAs regulates tumour biology. Nature. 2010;465(7301):1033-8. https://doi.org/10.1038/na ture09144.

25. Bosson AD, Zamudio JR, Sharp PA. Endogenous miRNA and target concentrations determine susceptibility to potential ceRNA competition. Mol Cell. 2014;56(3):347-59. https://doi.org/10.1016/j.molcel.2014.09.018.

26. Denzler R, Agarwal V, Stefano J, Bartel DP, Stoffel M. Assessing the ceRNA hypothesis with quantitative measurements of miRNA and target abundance. Mol Cell. 2014;54(5):766-76. https://doi.org/10.1016/j.molcel.2 014.03.045.

27. Gocheva V, Wang HW, Gadea BB, Shree T, Hunter KE, Garfall AL, et al. IL-4 induces cathepsin protease activity in tumor-associated macrophages to promote cancer growth and invasion. Genes Dev. 2010;24(3):241-55. https://doi.org/10.1101/gad.1874010.

28. Petermann I, Mayer C, Stypmann J, Biniossek ML, Tobin DJ, Engelen MA et al. Lysosomal, cytoskeletal, and metabolic alterations in cardiomyopathy of cathepsin L knockout mice. FASEB J. 2006;20(8):1266-8. https://doi.org/1 0.1096/fj.05-5517fje.

29. Sun M, Ouzounian M, de Couto G, Chen M, Yan R, Fukuoka M, et al. Cathepsin-L ameliorates cardiac hypertrophy through activation of the autophagy-lysosomal dependent protein processing pathways. J Am Heart Assoc. 2013;2(2):e000191. https://doi.org/10.1161/JAHA.113.000191.

30. Olson OC, Joyce JA. Cysteine cathepsin proteases: regulators of cancer progression and therapeutic response. Nat Rev Cancer. 2015;15(12):712-29. https://doi.org/10.1038/nrc4027.

31. Dana D, Pathak SK. A review of small molecule inhibitors and functional probes of human cathepsin L. Molecules. 2020;25(3):698-735.https://doi. org/10.3390/molecules 25030698

\section{Publisher's Note}

Springer Nature remains neutral with regard to jurisdictional claims in published maps and institutional affiliations.

Ready to submit your research? Choose BMC and benefit from:

- fast, convenient online submission

- thorough peer review by experienced researchers in your field

- rapid publication on acceptance

- support for research data, including large and complex data types

- gold Open Access which fosters wider collaboration and increased citations

- maximum visibility for your research: over $100 \mathrm{M}$ website views per year

At $\mathrm{BMC}$, research is always in progress.

Learn more biomedcentral.com/submission 